\title{
Adaptation of the suprahyoid muscle complex to mandibular advancement surgery
}

\author{
David S. Carlson, Ph.D., Edward Ellis III, D.D.S., M.S., ${ }^{\star \star}$ and Paul C. Dechow, Ph.D. ${ }^{\star \star \star}$ \\ Ann Arbor, Mich., and Dallas, Texas
}

\begin{abstract}
The suprahyoid musculature has been implicated as one of the major factors responsible for relapse after mandibular advancement surgery. Previous studies have also indicated that the muscle and connective tissues comprising the suprahyoid complex must adapt to increased length brought about by mandibular advancement for skeletal stability to be achieved. The purpose of this study was to provide quantitative data concerning the immediate changes and long-term adaptations that take place within the suprahyoid complex over a 2-year period after mandibular advancement. Mandibular advancement was performed on ten adult Macaca mulatta monkeys with and without suprahyoid myotomy ( $n=5$ /group). Six animals were used as controls. Mandibular length and changes in the length of the various anatomic regions of the suprahyoid complex were evaluated radiographically with the aid of radiopaque bone, muscie, and tendon markers implanted preoperatively. The results for the nonmyotomy group showed that (1) the suprahyoid complex was elongated approximately two thirds the amount of mandibular lengthening, (2) the major immediate adaptations within the suprahyoid complex after the surgical procedure occurred at the muscle-bone interface and the muscle-tendon interface, (3) the change in length at the muscle-tendon junction was maintained throughout the 2-year follow-up period, indicating that significant long-term adaptations took place primarily at that location, and (4) no significant short-term changes or long-term adaptations were seen within the anterior digastric muscle or the intermediate digastric tendon. Within the myotomy group, it was found that (1) the suprahyoid complex recoiled immediately after myotomy such that the anterior belly of the digastric muscle became separated from the advanced distal mandibular segment by more than twice the amount of mandibular lengthening, (2) the anterior digastric muscle remained essentially at this posterior position throughout the 2-year follow-up period, and (3) though not significant, there was a trend for a decrease in the length of the anterior digastric muscle belly. On the basis of these results, it was concluded that both short-term changes and long-term adaptations to lengthening of the suprahyoid complex as a result of mandibular lengthening occur primarily within the connective tissues comprising the muscle-tendon and muscle-bone interfaces, not within the muscle fibers themselves. (AM J ORTHOD DENTOFAC ORTHOP 1987;92:134-43.)
\end{abstract}

\begin{abstract}
$\mathrm{T}$ he suprahyoid musculature has repeatedly been suggested as a primary cause of relapse after mandibular advancement surgery. ${ }^{1-9}$ Based on clinical investigations, it has been hypothesized that when lengthened the suprahyoid musculature exerts posteriorly directed forces on the advanced mandible as a result of active muscle contraction, recoil of stretched elastic connective tissue elements, or both. In addition, a recent experimental study in our laboratory demonstrated that the suprahyoid muscle complex is one of the major
\end{abstract}

*Associate Professor, Departments of Orthodontics and Anatomy \& Cell Biology; Associate Research Scientist, Center for Human Growth and Development, The University of Michigan.

**Assistant Professor, Department of Oral \& Maxillofacial Surgery; Research Investigator, Center for Human Growth and Development, The University of Michigan.

***Assistant Professor, Department of Gross Anatomy, Baylor College of Dentistry. precipitating factors in relapse after mandibular advancement. ${ }^{10,11}$

There is some indirect clinical and experimental evidence to support the hypothesis that stretch of the suprahyoid complex is a primary factor leading to skeletal relapse after mandibular advancement (see Carlson and associates, ${ }^{10}$ and Ellis and Carlson ${ }^{11}$ for reviews). However, there is no direct empirical evidence concerning what happens within the suprahyoid muscle complex after mandibular advancement. For example, there are no empirical data indicating (1) whether or not the suprahyoid muscle complex is actually stretched as an immediate result of mandibular advancement, (2) if it is, where and to what extent it is stretched, and (3) the degree to which any immediate stretch that does occur is maintained as a permanent adaptation to altered mandibular length. There are also no quantitative data concerning the fate of the suprahyoid muscle complex 
after it is detached during mandibular advancement osteotomy. ${ }^{11.12}$

The purpose of this article is to provide quantitative data concerning short-term change and long-term (2 years postsurgery) adaptation of the suprahyoid muscle complex after mandibular advancement surgery with and without suprahyoid myotomy in a controlled experimental study. This article thus completes the cephalometric component of a long-term experimental study of relapse and musculotendinous adaptation after mandibular advancement, the first part of which dealt with skeletal relapse after mandibular advancement with and without suprahyoid myotomy. ${ }^{11}$

\section{MATERIALS AND METHODS Experimental animals}

Ten adult rhesus monkeys (Macaca mulatta), nine females and one male, were used in this experiment. An additional six adult monkeys, three males and three females, were available for control data. On the basis of tooth eruption scales, each of the animals was at least 4 to $4 \frac{1}{2}$ years old at the beginning of the experiment.

The ten experimental animals were divided into two groups, both of which underwent mandibular advancement surgery to produce a 4 to $6-\mathrm{mm}$ lengthening of the mandible. Animals in group MAA (mandibular advancement--attached) had the advancement with the suprahyoid muscles left intact. Animals in group MAD (mandibular advancement-detached) underwent a suprahyoid myotomy as part of the surgical procedure.

\section{Bone implant techniques}

Sterile tantalum bone implants were placed aseptically in the craniofacial complex of the experimental animals according to our standard protocols. ${ }^{10}$ Five to six implants were placed in the mandible, three in the maxilla, one in the frontal bone, and three in the sphenoid region of the cranial base.

\section{Muscle-tendon implants}

Muscle and tendon markers consisting of short pieces of gold root canal broach were used to evaluate the position of the various components of the suprahyoid complex radiographically. Muhl ${ }^{13}$ and Muhl and Grimm $^{14}$ showed that these markers remain stable when implanted into muscle and allow accurate radiographic evaluation of spatial position. Several other studies from our laboratories have demonstrated the utility of these muscle implants as radiopaque markers for use in longitudinal cephalometric analyses of musculotendinous growth, migration, and adaptation. ${ }^{10,15,16}$

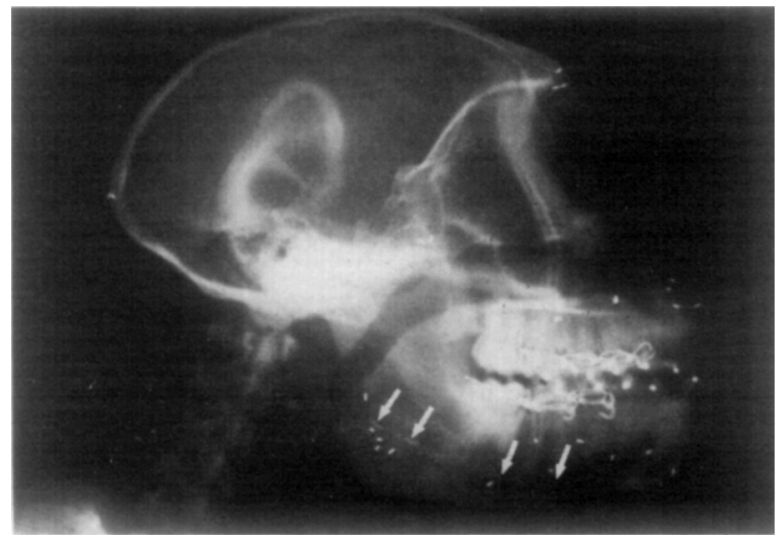

Fig. 1. Lateral radiographic cephalogram of a female rhesus monkey indicating the positions of the radiopaque markers within the anterior belly of the digastric muscle and tendon markers within the intermediate digastric tendon.

Under aseptic conditions and general anesthesia, a $3-\mathrm{cm}$ incision was made through skin and subcutaneous tissues from the lingual aspect of the mandibular symphysis to the hyoid bone. The platysma was dissected to expose the anterior belly of the digastric muscle and the intermediate tendon of the digastric complex. Four short pieces of gold barbed root canal broach were injected individually through an 18-gauge needle into the suprahyoid complex. Two were placed longitudinally into the intermediate tendon of the digastric complex and two were longitudinally placed into the anterior digastric muscle (Fig. 1). Serial lateral radiographic cephalograms were obtained on all animals before initiation of the experiment to establish that the implants were stable. Only after the muscle markers were considered stable, usually within 6 to 8 weeks, were the animals considered ready for the surgery. Serial cephalometric analyses of the muscle and tendon implants in the control animals indicated that the muscle implants moved an average of no more than $0.2 \mathrm{~mm}$ and that tendon implants moved an average of no more than $0.05 \mathrm{~mm}$ in any direction over a 6-month period.

\section{Mandibular advancement surgical procedure}

Mandibular advancements were achieved by means of a standard C-form osteotomy with only minor modification for use with rhesus monkeys. In group MAA the suprahyoid muscles were left intact. In group MAD the anterior digastric and geniohyoid muscles were sharp dissected from the lingual aspect of the mandible and allowed to retract. This procedure was described in detail in recent articles dealing with skeletal relapse after mandibular advancement. ${ }^{10.11}$ 


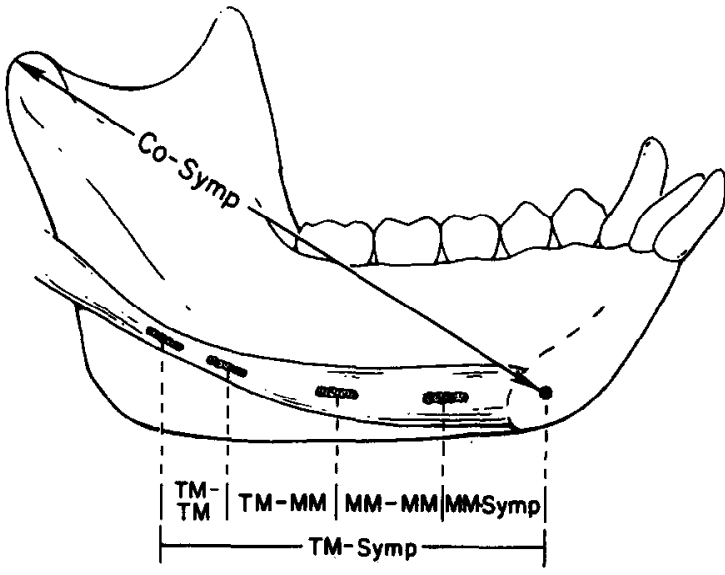

Fig. 2. Diagram indicating the six linear measurements that were used to determine serial changes in mandibular length and within the suprahyoid complex. C, condylion; SYMP, symphyseal bone marker; $M M$, muscle markers; $T M$, tendon markers. (1) Mandibular length was determined by measuring from a bone marker in the mandibular symphysis to condylion, the most posterior-superior point along the condyle. (2) Adaptations at the muscle-bone interface were determined by measuring from the symphyseal bone marker to the most anterior muscle implant. (3) Adaptation within the anterior digastric muscle was determined by measuring from the most anterior to the most posterior muscle implants. (4) Adaptation at the muscletendon interface was determined by measuring between the most posterior muscle implant and the most anterior tendon implant. (5) Adaptation within the intermediate tendon of the digastric muscle was determined by measuring between the most anterior and most posterior tendon implants. (6) Adaptation within the total suprahyoid complex was determined by measuring from the symphyseal bone marker to the most distal tendon implant.

\section{Analytic techniques}

Lateral radiographic cephalograms of each animal were taken immediately preoperatively, immediately postoperatively, 6 weeks postoperatively, upon release of maxillomandibular fixation (immediately after the 6week postoperative x-ray film), and $12,24,48$, and 96 weeks thereafter.

Each radiographic cephalogram was traced according to our standard protocols, ${ }^{10}$ taking into account the muscle and tendon implants in the suprahyoid region. The serial tracings were then digitized and the coordinate data inputted into the computer system at The University of Michigan for quantitative analysis.

For purposes of analysis, the suprahyoid muscle complex was partitioned into four separate anatomic sites-the muscle-bone interface, the belly of the anterior digastric muscle, the muscle-tendon interface, and the intermediate tendon of the digastric muscle. Six linear cephalometric variables were calculated at each
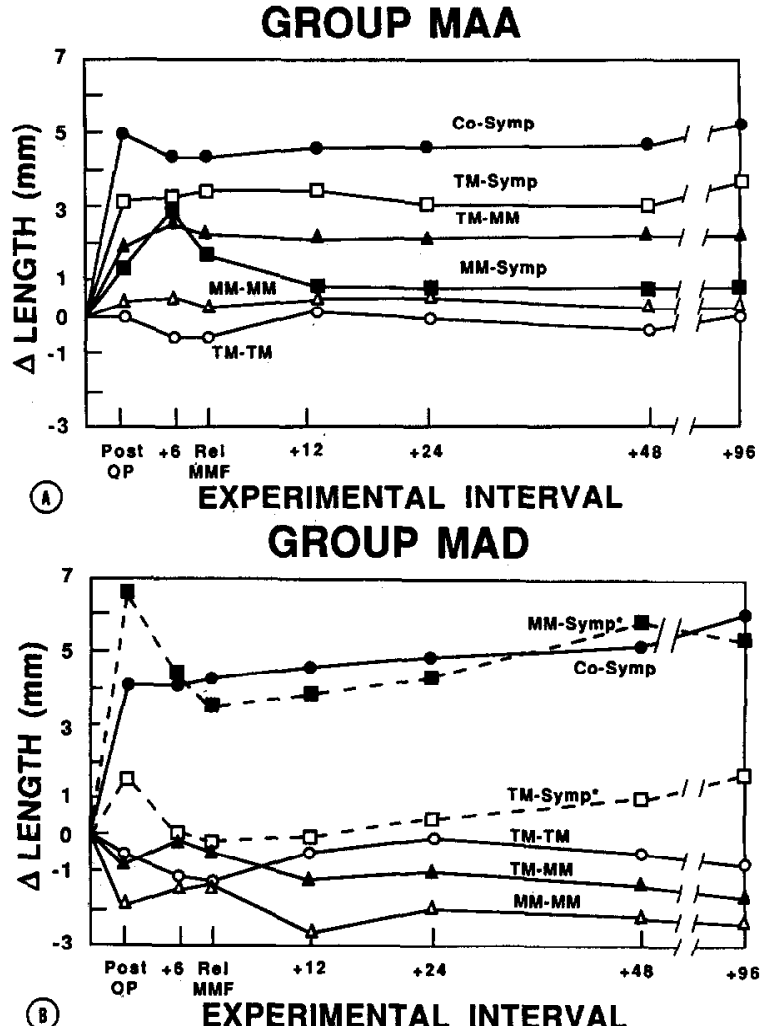

Flg. 3. Graph of mean serial changes in each of the cephalometric variables over a 96-week experimental period for group MAA (A) and group MAD (B). The values for the muscle-bone interface length (MM-SYMP) and total suprahyoid complex length (TM-SYMP) in $\mathbf{B}$ indicate those lengths minus the amount of mandibular advancement in order to demonstrate the amount of change in the suprahyoid complex relative to its preoperative length.

interval for each animal corresponding to these four anatomic sites (Fig. 2).

Serial changes in each of these variables were calculated throughout the 96-week postfixation period. The data were considered in terms of change relative to their preoperative length and, for data from group MAA animals, their contribution to the total mandibular advancement. Multivariate one-way analysis of variance was used to detect significant changes between each of the serial time intervals. Statistical significance indicated that the change between serial intervals was significantly different from zero. An immediate change in any of the variables other than mandibular length was taken to indicate stretch (group MAA) or recoil (group MAD) of the soft tissues that may or may not be maintained as a permanent adaptation. Maintenance of a change in each of these variables over time was considered to be evidence of a long-term adaptationprobably caused by growth leading to increased length 
Table I. Adaptation within the suprahyoid muscle complex during a 96-week period after mandibular advancement without suprahyoid myotomy (group MAA) (mean values identify differences in mean length to the preoperative value $[\mathrm{N}=5]$ )

\begin{tabular}{|c|c|c|c|c|c|c|c|}
\hline & $\begin{array}{c}\text { Immediate } \\
\text { postop }\end{array}$ & $\begin{array}{c}6 w k \\
\text { postop }\end{array}$ & $\begin{array}{c}\text { Release } \\
\text { of } \\
M M F\end{array}$ & $\begin{array}{c}12 w k \\
\text { post-MMF }\end{array}$ & $\begin{array}{c}24 w k \\
\text { post-MMF }\end{array}$ & $\begin{array}{c}48 w k \\
\text { post-MMF }\end{array}$ & $\begin{array}{c}96 w k \\
\text { post-MMF }\end{array}$ \\
\hline \multicolumn{8}{|c|}{ 1. Mandibular length (C-Symp) } \\
\hline$\overline{\mathrm{x}}$ & $5.03^{* *}$ & $4.37^{*}$ & 4.40 & 4.64 & 4.77 & 4.89 & 5.28 \\
\hline SD & 0.44 & 0.38 & 0.38 & 0.47 & 0.49 & 0.47 & 0.50 \\
\hline \multicolumn{8}{|c|}{ 2. Muscle-bone interface (Symp-MM) } \\
\hline$\overline{\mathrm{x}}$ & $1.30^{*}$ & $3.11^{* *}$ & 1.67 & 0.88 & 0.76 & 0.75 & 0.73 \\
\hline $\mathrm{SD}$ & 0.25 & 0.61 & 0.30 & 0.20 & 0.14 & 0.13 & 0.15 \\
\hline \multicolumn{8}{|c|}{ 3. Within muscle (MM-MM) } \\
\hline$\overline{\mathrm{x}}$ & 0.42 & 0.35 & 0.19 & 0.46 & 0.53 & 0.56 & 0.55 \\
\hline SD & 0.11 & 0.08 & 0.05 & 0.15 & 0.16 & 0.18 & 0.17 \\
\hline \multicolumn{8}{|c|}{ 4. Muscle-tendon interface (MM-TM) } \\
\hline $\bar{x}$ & $1.89^{* *}$ & $2.81 * *$ & 2.16 & 2.05 & 2.23 & 2.29 & 2.08 \\
\hline $\mathrm{SD}$ & 0.58 & 0.84 & 0.64 & 0.63 & 0.69 & 0.66 & 0.63 \\
\hline \multicolumn{8}{|c|}{ 5. Within tendon (TM-TM) } \\
\hline $\bar{x}$ & 0.00 & -0.52 & -0.47 & 0.40 & 0.00 & -0.14 & 0.44 \\
\hline SD & 0.26 & 0.13 & 0.16 & 0.14 & 0.14 & 0.15 & 0.15 \\
\hline \multicolumn{8}{|c|}{ 6. Total suprahyoid complex (Symp-TM) } \\
\hline$\overline{\mathrm{x}}$ & $2.11^{*}$ & 3.24 & 3.42 & 3.39 & 3.18 & 3.26 & 3.71 \\
\hline SD & 0.16 & 0.57 & 0.58 & 0.59 & 0.55 & 0.56 & 0.60 \\
\hline
\end{tabular}

${ }^{*} P<0.05 ;{ }^{*} P<0.01$. Statistical significance indicates that the change from one interval to the next was significantly different from zero.

or remodeling resulting in decreased length—at each region.

\section{RESULTS}

The results for each of the variables are presented in Tables I and II, and are summarized graphically in Fig. 3.

\section{Mandibular length}

Mandibular length in group MAA animals increased an average of $5.03 \mathrm{~mm} \mathrm{(7 \% )}$ as a result of the osteotomy. By the sixth week postoperative, the mean mandibular length hacl decreased by $0.66 \mathrm{~mm}$ ( $13 \%$ relapse; $P<0.01)$. There were no significant changes in mandibular length after the period of fixation.

Mandibular length in group MAD animals increased an average of $4.18 \mathrm{~mm}(6 \%)$ as a result of the osteotomy. There was no decrease in mandibular length in any animal in this group during the period of maxillomandibular fixation or at any time thereafter. In fact, by the 96-week interval, there was a slight but statistically significant $(P<0.03)$ increase in mandibular length.

\section{Muscle-bone interface}

Immediately after the surgical procedure in group MAA, there was a significant $(P<0.05)$ increase in the distance between the symphyseal bone marker and the most anterior muscle implant. Although this represented only a $5 \%$ increase in length at the musclebone interface, taken as a proportion of the total amount of mandibular lengthening, it accounted for nearly $25 \%$ of the mandibular advancement. By 6-weeks postoperative, the distance at the muscle bone interface had increased significantly $(P<0.05)$ once again; however, this appeared to be a transient phenomenon because by 12 weeks it had returned to between the preoperative and the immediate postoperative lengths. By 96 weeks postoperative, adaptations at the muscle-bone interface accounted for an increase in length of less than $1 \mathrm{~mm}$.

Change at the muscle-bone interface immediately after the surgical procedure in group MAD was obviously an artifact of the surgical detachment itself. Immediately after mandibular advancement, the anterior belly of the digastic muscle became separated from the bone by an average of $11.49 \mathrm{~mm}$, more than double the average amount of mandibular lengthening (4.18 $\mathrm{mm}$ ). There was no indication at the time of the surgical procedure that this retraction was due to active muscle contraction, particularly since the animals were at a surgical level of anesthesia at the time the muscles recoiled and at the time they were radiographed. This result provides good evidence to indicate that the su- 
Table II. Adaptation within the suprahyoid muscle complex during a 96-week period after mandibular advancement with suprahyoid myotomy (group MAD) (mean values indicate differences in mean length relative to the preoperative value $[\mathrm{N}=5]$ )

\begin{tabular}{|c|c|c|c|c|c|c|c|}
\hline - & $\begin{array}{c}\text { Immediate } \\
\text { postop }\end{array}$ & $\begin{array}{c}6 w k \\
\text { postop }\end{array}$ & $\begin{array}{c}\text { Release } \\
\text { of } \\
M M F\end{array}$ & $\begin{array}{c}12 w k \\
\text { post-MMF }\end{array}$ & $\begin{array}{c}24 w k \\
\text { post-MMF }\end{array}$ & $\begin{array}{c}48 w k \\
\text { post-MMF }\end{array}$ & $\begin{array}{c}96 w k \\
\text { post-MMF }\end{array}$ \\
\hline \multicolumn{8}{|c|}{ 1. Mandibular length (C-Symp) } \\
\hline$\overline{\mathbf{x}}$ & $4.19 * *$ & 4.19 & 4.31 & 4.51 & 4.67 & 5.14 & $5.95^{*}$ \\
\hline SD & 0.21 & 0.17 & 0.19 & 0.16 & 0.16 & 0.19 & 0.30 \\
\hline \multicolumn{8}{|c|}{ 2. Muscle-bone interface (Symp-MM) } \\
\hline$\overline{\mathbf{x}}$ & $11.49 * *$ & $8.57 * *$ & 7.76 & 8.08 & 8.61 & 9.03 & 9.49 \\
\hline SD & 1.03 & 0.65 & 0.65 & 0.52 & 0.59 & 0.70 & 0.77 \\
\hline \multicolumn{8}{|c|}{ 3. Within muscle $(M M-M M)$} \\
\hline$\overline{\mathbf{x}}$ & $-2.01 *$ & -1.49 & -1.43 & -1.93 & -2.73 & -2.32 & -2.55 \\
\hline SD & 0.40 & 0.21 & 0.22 & 0.38 & 0.34 & 0.52 & 0.85 \\
\hline \multicolumn{8}{|c|}{ 4. Muscle-tendon interface (MM-TM) } \\
\hline $\bar{x}$ & -0.83 & -0.03 & -0.28 & -1.19 & -1.05 & -1.42 & -1.53 \\
\hline SD & 0.28 & 0.27 & 0.23 & 0.39 & 0.35 & 0.52 & 0.55 \\
\hline \multicolumn{8}{|c|}{ 5. Within tendon (TM-IM) } \\
\hline$\overline{\mathbf{x}}$ & -0.55 & -1.19 & -1.33 & -0.41 & -0.11 & -0.43 & -0.61 \\
\hline SD & 0.46 & 0.71 & 0.68 & 0.32 & 0.08 & 0.29 & 0.53 \\
\hline \multicolumn{8}{|c|}{ 6. Total suprahyoid complex (Symp-TM) } \\
\hline$\overline{\mathrm{x}}$ & $5.75^{* *}$ & $4.24 *$ & 4.09 & 4.15 & 4.74 & 5.19 & 4.95 \\
\hline SD & 0.48 & 0.25 & 0.77 & 0.33 & 0.36 & 0.35 & 0.43 \\
\hline
\end{tabular}

${ }^{*} P<0.05 ; * * P<0.01$. Statistical significance indicates that the change from one interval to the next is significantly different from zero.

prahyoid muscle complex is under some degree of tension due to elastic connective tissues within the muscle complex and that this tension is capable of expressing itself by a posteriorly directed pull on the anterior portion of the mandible. Within 6 weeks after surgical treatment, there was a tendency for the anterior digastric muscle to move anteriorly relative to the mandibular symphysis to some degree; it remained there throughout the 96-week experimental period.

\section{Anterior digastric muscle}

There were no significant changes in this length at any time during the experimental period in group MAA animals. Three of the five MAA animals did experience a marked increase in the length of the anterior digastric muscle immediately after the surgical procedure; however, this appeared to be a transient phenomenon as all but one animal returned to and remained at very near preoperative values by 12 weeks after surgery.

In the group MAD animals, there was a significant amount of change within the anterior belly of the digastric muscle immediately after surgery as the two implants in the belly of the muscle moved closer together. This was undoubtedly caused by recoil of the elastic connective tissue elements within the muscle itself. The muscle markers maintained essentially the same shortened distance from each other throughout the rest of the experimental period.

\section{Muscle-tendon interface}

As an immediate result of surgical treatment, the distance between the most posterior muscle implant and the most anterior tendon implant increased $11 \%$ $(P<0.05)$ in group MAA animals, which accounted for $36 \%$ of the total mandibular advancement. The distance between the muscle and tendon implants continued to increase $(P<0.01)$ through the 6-week interval so that by the 96-week interval it was $12 \%$ longer than it was before the surgical procedure. This accounted for an average of $38 \%$ of the total amount of mandibular advancement. There was no significant change at the muscle-tendon interface in group MAD animals.

\section{Intermediate tendon}

There were no indications of any changes in the length of the intermediate tendon as a result of mandibular advancement either immediately after surgical treatment or at any time throughout the experimental period in group MAA and group MAD animals.

\section{Total suprahyold complex}

Change in the length of the total suprahyoid complex in group MAA after the surgical procedure was indicative of the amount of soft-tissue stretch that occurs anterior to the hyoid bone in response to mandibular advancement. Immediately after surgery, the suprahyoid complex increased in length by an average of 
$3.11 \mathrm{~mm}$ or $62 \%$ of the total mandibular advancement $(P<0.05)$. This constituted an average $7 \%$ increase in overall length relative to the preoperative condition. By the end of the experimental period, the suprahyoid complex in group MAA had increased in length by an average of $3.71 \mathrm{~mm}$ or $71 \%$ of the total advancement. Thus, the initial stretch and subsequent long-term adaptation of the suprahyoid muscle complex anterior to the hyoid bone accounted for approximately two thirds of the total soft-tissue adaptation, leaving the remaining one third of the change in length to be accounted for by the posthyoid musculature (that is, posterior digastric and infrahyoid muscles).

In group MA.D, release of the suprahyoid muscles at the time of surgical treatment resulted in an average increase in the clistance between the posterior tendon marker and the mandibular symphysis of $5.75 \mathrm{~mm}$, which was only slightly greater than the average amount of skeletal advancement. Therefore, it can be reasoned that there was only a small recoil of the total suprahyoid complex as a whole immediately after detachment of the suprahyoid muscles. By 6 weeks postoperative, however, this recoil had been essentially eliminated altogether and the intermediate tendon was at its original spatial position relative to the overall suprahyoid region.

\section{DISCUSSION}

The results of this study demonstrate that the total suprahyoid muscle complex was lengthened as a result of mandibular advancement without suprahyoid myotomy. As an irnmediate result of the surgical procedure, the entire suprahyoid muscle complex was brought forward approximately two thirds of the total amount of mandibular lengthening. This was accomplished primarily by stretch at the muscle-bone interface and at the muscle-tendon interface. There were no significant changes in length within the anterior belly of the digastric muscle and within the intermediate digastric tendon. The remaining one third of the immediate stretch brought about by the mandibular lengthening must have taken place in the region distal to the anterior digastric muscle-tendon complex, that is, in the posterior digastric and infrahyoid complexes. By 2 years after surgery, the suprahyoid muscle complex accounted for approximately one half of the permanent adaptations in muscle-tendon length associated with the skeletal advancement without suprahyoid myotomy.

Immediately after mandibular advancement with suprahyoid myotomy, the suprahyoid complex underwent a slight recoil relative to its original position. There were no other significant immediate or long-term adaptations within the total suprahyoid complex or at each anatomic site within this complex over the remainder of the 2-year study period.

Previous publications from our laboratory put forward the concept that adaptation to altered length of the muscles of mastication as a result of orthognathic surgery could occur at several different anatomic locations including (1) within bone or at bony articulations, which would be defined as "relapse," (2) at the muscle-bone interface, (3) within muscle, (4) at the muscle-tendon interface, and (5) within tendon. ${ }^{1 n .18}$ While adaptation at each of these sites is possible, however, each site may respond quite differently to an alteration in skeletal form that results in a lengthening of the associated muscle complex. In terms of the present study, not all regions within the suprahyoid complex were stretched equally as an immediate result of mandibular advancement without suprahyoid myotomy; nor did all of the regions of the suprahyoid complex respond in an equivalent manner during the 2-year period after the surgical procedure. Adaptation within bone and at bony articulations was discussed in our previous article on skeletal relapse after mandibular advancement with and without suprahyoid myotomy. The remainder of this discussion will focus on musculotendinous adaptations.

\section{Adaptation at the muscle-bone and muscle-tendon interfaces}

The muscle-bone interface is characterized by the presence of Sharpey's fibers or collagenous elements of the muscle that continue directly into the fibrous layer of periosteum or into the bone itself. ${ }^{19-21}$ Experimental studies have shown that growth at the muscle-bone interface occurs normally during development, probably caused by both an increase in muscle length through the addition of scrial sarcomeres and remodeling of the collagenous interface. ${ }^{10,15,21,22}$ Similarly, at the muscletendon interface, collagenous fibrils run from the muscle sarcolemma, perimysium, and epimysium into the tendon, where they become interdigitated with the fibers comprising the tendon itself. ${ }^{20.23}$

Experimental studies have shown that it is primarily at the attachment sites of muscle that normal growthrelated adaptations and adaptations to increased muscle length take place. ${ }^{24-27}$ This is supported by the results of the present study. Specifically, it is most likely that short-term changes at the muscle-bone and muscletendon interfaces as a result of mandibular advancement without myotomy were due to stretch of the connective tissues forming the connection with the muscle fibers. The fact that the immediate changes at the muscletendon interface were maintained over the long-term follow-up period of nearly 2 years indicates that they 
became incorporated as permanent adaptations, probably as a result of growth of the collagenous fibrils that connect the terminal end of the muscle and the intermediate tendon.

\section{Adaptation within the anterior digastric muscle}

There are numerous related adaptations that can take place within muscle in response to an increase in muscle length. Immediately after muscle lengthening, two specific morphologic changes take place. First, the parallel and serial connective tissues within the muscle become stretched. Second, once the connective tissues have reached the limit of their extensibility, the muscle fibers themselves become stretched, resulting in an elongation of the sarcomeres and a decrease in the overlap of actin and myosin filaments. The net effect of stretching of the connective tissue with or without muscle fiber stretch is an increase in the passive tension generated by the connective tissues. In addition, the presence of stretch receptors within the muscle and connective tissue will likely result in active contraction of the muscle fibers until either the resting length of the muscle is restored or the bias of the stretch receptors accommodates to the increased muscle length. A second mechanism intrinsic to the muscle for reestablishing sarcomere length after stretch involves the addition of sarcomeres within each fiber, which results in a lengthening of the muscle fibers while at the same time reducing the contribution that each sarcomere must make to the overall length of the fiber. Increases in fiber length caused by addition of sarcomeres take place during normal growth and in response to surgically induced changes in muscle length and muscle function. ${ }^{24.27,28}$ The converse, a decrease in fiber length caused by a decrease in the number of serial sarcomeres, takes place when muscles are maintained chronically at a shortened length. $^{22}$

Contrary to our original expectations, there was no evidence of any "stretch" of the anterior belly of the digastric muscle immediately after mandibular advancement without myotomy. Nor was there any evidence of significant lengthening of the muscle at any time thereafter over a 2-year postoperative period. As the radiopaque muscle implants reside within the perimysium, not within the fibers themselves, this finding indicates not only that the muscle fibers themselves were not stretched but also that the parallel connective tissue elements within the muscle belly were not stretched significantly as a result of mandibular advancement.

This observation is supported by data from related studies of the fibers comprising the anterior belly of the digastric muscle after mandibular advancement with and without suprahyoid myotomy in our laboratory. ${ }^{27,29}$
Histochemical analysis of myofibrillar ATPase and oxidative enzymes (SDH), and biochemical analysis of succinate oxidase activity failed to indicate any change in the function of the anterior digastric muscle that could be attributed to the lengthening of the muscle as a result of surgical treatment 1 year earlier. Similarly, histologic analysis of fiber cross-sectional area failed to indicate any change in muscle function. There were also no significant differences in the proportion of fiber types, oxidative capacity, and cross-sectional area of the digastric muscle after mandibular advancement with suprahyoid myotomy. Although this finding was somewhat unexpected, particularly in terms of fiber crosssectional area, it should be noted that the data in that study describe the condition of the muscle 1 year after the surgical procedure and do not necessarily indicate short-term adaptations.

In a study of short-term muscle adaptation, ${ }^{27}$ histologic analysis of the length of individual fibers comprising the digastric muscle and of the sarcomeres within these fibers showed that there were no differences from control values at any time within a 16-week period after mandibular advancement surgery without suprahyoid myotomy. Detachment of the suprahyoid muscles at the time of surgical treatment, however, resulted in a decrease in the length of the fibers as a result of the loss of serial sarcomeres. These fibers remained significantly shorter than normal for the duration of the experimental period. This finding supports the cephalometric results reported in the current study and the morphologic evidence reported earlier ${ }^{11}$ in which it was found that there was a significant amount of scar tissue that had become organized into a broad "tendon" connecting the shortened anterior belly of the digastric muscle with the advanced mandibular symphysis in group MAD animals.

These results and those of the present study clearly indicate that suprahyoid muscles do not experience any significant amount of stretch as a result of mandibular advancement. Combined with the fact that the anterior digastric muscle has a paucity of muscle spindles, ${ }^{30}$ it is therefore extremely unlikely (1) that there was any reflex-mediated activity induced by stretch of the anterior belly of the digastric and the geniohyoid muscles that could contribute to relapse tendencies, and (2) that growth-related adaptations-that is, addition of sarcomeres within fibers and corresponding lengthening of the fibers and of the whole muscle-take place as a result of mandibular advancement.

\section{Role of the suprahyoid complex in relapse}

Various methods to accommodate or alleviate the presumed distracting forces produced by the suprahyoid 
- muscle complex have been advocated. Each of these methods is predicated on the assumption that the suprahyoid muscles and associated soft tissues are largely responsible for exerting posteriorly directed forces on the advanced mandibular segment.

Surgical overcorrection. Surgical overcorrection of the mandible-that is, advancement of the distal mandibular segment beyond the desired length in the anticipation of relapse - was a relatively common method to deal with the potential of relapse. ${ }^{1,2,12,31}$ It is particularly difficult, however, to predict the amount of relapse that might occur after mandibular advancement. In fact, there may be little or no relapse in many cases, which would result in a mandible that is longer than is desirable if overcorrection is done. Moreover, since the amount of advancement may be a factor in relapse, ${ }^{32}$ overcorrection may actually contribute to relapse in some cases. Overcorrection of the mandible is also not appealing because it asserts a belief in the inevitability of relapse and professes an inability to control the factors responsible for relapse clinically.

Orthopedic braces. Use of shoulder-chin braces or cervical collars to alleviate relapse tendencies after mandibular advancement is also common. ${ }^{1-3,7,9,31}$ Dealing directly with the presumed cause of relapse of the advanced mandible, as this approach does, it is clearly more appealing conceptually than overcorrection. Specifically, the purpose of using a shoulder-chin brace or cervical collar after mandibular advancement surgery is to maintain the distal mandibular segment in an anterior and superior position physically until the soft tissues, such as the muscles and connective tissues of the suprahyoid muscle complex, adapt to the altered mandibular length and any adverse tension that might produce relapse is alleviated. Drawbacks of this approach include the fact that the amount of time necessary for the brace and collar to be worn while the muscles and soft tissues adapt to the altered mandibular length is unknown. In addition, clinical studies have shown that significant relapse can still occur during the period of maxillomandibular fixation, despite the use of a shoulder-chin brace or cervical collar. ${ }^{2.9}$ This approach has the additional drawback of requiring patients to wear bulky braces for extended periods of time.

Suprahyoid myotomy. The suprahyoid myotomy would seem to be the most direct method to obviate the tension within the suprahyoid complex after the mandible is lengthened..$^{5,11,12}$ Like the use of a shoulderchin brace and cervical collar, the suprahyoid myotomy deals directly with the presumed cause of relapse after mandibular advancement, seeking to alleviate altogether the muscle and soft-tissue forces that cause the advanced mandibular segment to be translated poste- riorly. A suprahyoid myotomy is relatively simple to accomplish surgically and is well tolerated by patients. For these reasons, it is one of the more common methods of attempting to reduce the tendency toward relapse after mandibular advancement.

On the basis of clinical impressions, Epker, Wolford, and Fish ${ }^{5}$ developed a model they believed would predict the amount of lengthening of the suprahyoid complex that occurs with mandibular advancement. Using this model in conjunction with information about basic muscle physiology and assumptions about the effect of mandibular advancement on the tissues comprising the suprahyoid complex, these investigators put forward recommendations concerning the amount of advancement that may be done without a suprahyoid myotomy. Specifically, they recommended that a suprahyoid myotomy be performed when the amount of the advancement would produce what they calculated to be a $15 \%$ stretch of the suprahyoid muscles beyond their resting length. Two assumptions of that model are that the hyoid bone is a stable structure and that all the stretch-lengthening of the suprahyoid complex takes place within the muscles. The estimate of how much muscle stretch can be tolerated during mandibular advancement was revised later to $30 \%$, and the more recent discussions of the model also tended to emphasize the importance of both connective tissue stretch and muscle stretch in skeletal relapse. ${ }^{33-35}$ There were no discussions of long-term adaptation of the suprahyoid complex to increased length.

Two specific features of the model developed by Epker, Wolford, and Fish are worthy of note. First, it has been shown cephalometrically that the hyoid bone frequently moves anteriorly as an immediate result of mandibular advancement surgery. ${ }^{2,6,36}$ Therefore, it is not appropriate to use the hyoid bone to determine the amount of lengthening that might occur in the suprahyoid region. Second, it is clear from the present study that the immediate stretch and long-term lengthening of the suprahyoid complex that occurs in association with mandibular advancement does not take place within the muscle, but is found primarily at the musclebone and muscle-tendon interfaces. This fact alone does not affect the general operation of the model proposed by Epker; however, it does indicate that one of the primary mechanisms that was proposed as being responsible for relapse after mandibular advancement, that is, muscle stretch and resultant active contraction, is not likely to occur. It would appear that soft-tissue stretch, particularly stretch of connective tissues at the muscle-bone and muscle-tendon interfaces, is considerably more important for both short-term and longterm adaptations in this regard. 


\section{CONCLUSION}

Our previous study of skeletal stability after mandibular advancement ${ }^{11}$ demonstrated that mandibular advancement without suprahyoid myotomy was associated with a statistically significant $13 \%$ relapse. There was no relapse after mandibular advancement with suprahyoid myotomy. On the basis of those results, it was concluded that the suprahyoid muscle complex does play a significant role in relapse after mandibular advancement. Because of the fact that skeletal relapse after mandibular advancement without myotomy occurred only during the period of maxillomandibular fixation, that is, the first 6 weeks, it was hypothesized that (1) the surgical procedure caused a stretching of the suprahyoid complex resulting in a posteriorly oriented force on the distal bone segment, and (2) this force was transitory in nature and became diminished over time as the suprahyoid muscle complex adapted to the initial stretch by permanently increasing in length.

The results of the present study support the above hypotheses. Although several components of the suprahyoid complex-including the muscle-bone interface, the muscle belly itself, and the muscle-tendon interface - were stretched to some degree as a result of the mandibular advancement, all but the stretch of the muscle-tendon interface were minimal. Moreover, only the muscle-tendon interface adapted by becoming permanently lengthened. Finally, both direct observation and cephalometric analysis indicated that there did not appear to be any detrimental effects of suprahyoid myotomy on the suprahyoid complex.

It is well known that when a muscle is lengthened the connective tissues associated with the muscle become stretched before any significant stretching and pulling apart of myofilaments within the muscle fibers themselves occur. ${ }^{10,16,22}$ The greater the amount of serial and parallel connective tissues associated with the muscle, the greater its extensibility before the muscle fibers are stretched. The results of the present study suggest, therefore, that lengthening of the mandible in the rhesus monkey approximately $7 \%$ was not sufficient to cause an initial stretch of the muscle fibers within the anterior belly of the digastric muscle as this amount of mandibular lengthening was accommodated by stretch of the connective tissues at the muscle-tendon and muscle-bone interfaces. A more substantial advancement or mandibular advancement in a subject with a more obtuse mandibular plane angle would be more likely to result in an initial stretch of the muscle itself because the greater the advancement, the greater the likelihood that the limits of extensibility of the connective tissues will be exceeded.

On the basis of the results of both our previous study of skeletal adaptations and of the present study of musculotendinous adaptations, the following conclusions of clinical relevance can be made. First, it is clear that in general the suprahyoid muscle complex plays a significant role in relapse after mandibular advancement surgery when the dentition is used to stabilize the advanced distal mandibular segment. However, the relative importance of the suprahyoid musculature in this regard will vary according to the specific case under consideration. A suprahyoid myotomy may be indicated in cases where several features-for example, a considerable amount of advancement and a high mandibular plane angle, among others-mitigate against stability after mandibular advancement. Second, release of the suprahyoid musculature and associated soft tissues from the advance mandibular segment via suprahyoid myotomy increases stability of the advanced mandibular segment and does not appear to have any recognizable detrimental effects on the suprahyoid muscle complex itself.

Perhaps the most rational alternative to performing a suprahyoid myotomy in an effort to increase skeletal stability is to use alternative methods of fixation of the skeletal segments. The results of this study clearly indicate that maxillomandibular fixation using the dentition is not a reliable means of holding the mandible forward until the segments have healed and the suprahyoid complex has adapted. If other means of fixation can be provided that prevent relapse and therefore provide the necessary time for adaptation of the suprahyoid complex and healing of the skeletal elements, nonrelapsing mandibular advancement surgery can be achieved. Skeletal suspension wires or rigid osseous fixation may provide this type of fixation.

Technical assistance on this study was provided by Dr. Emet Schneiderman, Vicki La Roche, and Donna Schimelfening. This research was supported by NIH Grants NIDR DE05232 and DE00109.

\section{REFERENCES}

1. Poulton DR, Ware WH. Surgical-orthodontic treatment of severe mandibular retrusion. AM J ORTHOD 1971;59:244-65.

2. Poulton DR, Ware WH. Surgical-orthodontic treatment of severe mandibular retrusion. Part II. AM J OkTHOD 1973;63:237-55.

3. McNeill RW, Hooley JR, Sundberg RJ. Skeletal relapse during intermaxillary fixation. J Oral Surg 1973;31:212-27.

4. Ive J, McNeill RW, West RA. Mandibular advancement: skeletal and dental changes during fixation. J Oral Surg 1977;35:881-6.

5. Epker BN, Wolford LM, Fish LC. Mandibular deficiency syndrome. Surgical considerations for mandibular advancement. Oral Surg Oral Med Oral Pathol 1978;45:349-63.

6. Kohn MW. Analysis of relapse after mandibular advancement surgery. J Oral Surg 1978;36:676-84.

7. Poulton DR, Ware WH, Baumrind S, Crane D. Surgical man- 
dibular advancement studied with computer-aided cephalometrics. AM J ORTHOD 1979;76:121-35.

8. Schendel SA, Wolford LM, Epker BN. Mandibular deficiency syndrome. III. Surgical advancement of the deficient mandible in growing children: treatment results in twelve patients. Oral Surg Oral Med Oral Pathol 1978;45:364-77.

9. Lake SL, McNeil RW, Little RM, West RA. Surgical mandibular advancement: a cephalometric analysis of treatment responses. AM J ORTHOD 1981;80:376-94.

10. Carlson DS, Ellis E III, Schneiderman ED, Ungerleider JC. Experimental models of surgical intervention in the growing face: cephalometric analysis of facial growth and relapse. In: McNamara JA Ji, Carlson DS, Ribbens KA, eds. The effect of surgical intervention on craniofacial growth. Monograph 12 , Craniofacial Growth Series. Ann Arbor: 1982;11-72. Center for Human Growth and Development, University of Michigan.

11. Ellis E III, Carlson DS. Stability two years after mandibular advancement surgery with and without suprahyoid myotomy: an experimental investigation. J Oral Maxillofac Surg 1983;41: 426-37.

12. Steinhauser EW. Advancement of the mandible by sagittal ramus split and suprahyoid myotomy. J Oral Surg 1973;31:516-21.

13. Muhl ZF. Longitudinal growth and response to change in length of the digastric rnuscle of the rabbit [Ph.D. Thesis]. Chicago: University of Illinois, 1974.

14. Muhl ZF, Grimm AF. Longitudinal growth of striated muscle; a radiographic study. Growth 1974;38:389-94.

15. Carlson DS. Growth of the masseter muscle in rhesus monkeys (Macaca mulatta). Am J Phys Anthropol 1983;60:401-10.

16. Carlson DS, Schneiderman ED. Cephalometric analysis of adaptations after lengthening the masseter muscle in adult rhesus monkeys. Arch Cral Biol 1983;28:627.

17. Schneiderman ED, Carlson DS. Cephalometric analysis of condylar adaptations to altered mandibular position in adult rhesus monkeys. Macaca mulatta. Arch Oral Biol 1985;30:49-54.

18. McNamara JA Jr, Carlson DS, Yellich GM, Hendricksoen RP. Musculoskeletal adaptation following orthognathic surgery. In: Carlson DS, MclNamara JA Jr, eds. Muscle adaptation in the craniofacial region. Monograph 8, Craniofacial Growth Series. Ann Arbor: 1978,91-132. Center for Human Growth and Development, University of Michigan.

19. Van der Klaauw CJ. Projections, deepening, and undulations of the surface of the skull in relation to the attachment of muscles. Verb Kou Ned Alsad Wetens Afk Naturk Ser 1963;2:1-247.

20. Moss ML, Moss-Salentijn L. The muscle-bone interface: An analysis of a morphological boundary. In: Carlson DS, McNamara JA Jr, eds. Muscle adaptation in the craniofacial region. Monograph 8, Craniofacial Growth Series. Ann Arbor: 1978; 39:71. Center for Human Growth and Development, University of Michigan.

21. Hoyte DAN, Enlow DH. Wolff's law and the problem of muscle attachment on resorptive surfaces of bone. Am J Phys Anthropol 1966;24:205-14.

22. Goldspink $G$. The adaptation of muscle to a new functional length. In: Anderson DJ, Matthews B, eds. Mastication. Bristol: John Wright \& Sons, Ltd, 1976;90-9.

23. Lanir Y. A microstructure model for the rheology of the mammalian tendon. Trans Am Soc Mech Eng 1980;102:332-9.

24. Crawford GNC. An experimental study of muscle growth in the rabbit. J Bone Joint Surg 1954;36:294-303.

25. Elliot DJ, Crawford GNC. The thickness and collagen content of tendon relative to the cross-sectional area of muscle during growth. Proc R Soc Lond 1965;162:198:202.

26. Grant PG, Hawes MR. Experimental modifications of muscle migration in the rabbit. J Anat 1977:123:361-7.

27. Carlson DS, Nemeth PA, Dechow PC. Fiber and sarcomere length in digastric muscles after mandibular advancement $[\mathrm{Ab}$ stract]. J Dent Res 1985;64:367.

28. Goldspink G, Williams PE. The nature of the increased passive resistance in muscle following immobilization of the mouse soleus muscle. J Physiol 1979;289:55.

29. Maxwell LC, Carlson DS, McNamara JA Jr, Faulkner JA. Effects of shortening and lengthening of mandible upon the char acteristics of masticatory muscle fibers in rhesus monkeys. In: Carlson DS, ed. Craniofacial biology. Monograph 10, Craniofacial Growth Series. Ann Arbor: 1980;121-40. Center for $\mathrm{Hu}-$ man Growth and Development, University of Michigan.

30. Dymytruk RJ. Neuromuscular spindles and depressor masticatory muscles of monkey. Am J Anat 1974;141:147-53

31. Guemsey L. Stability of treatment in Class II malocclusion corrected by full mandibular advancement surgery. Oral Surg Oral Med Oral Pathol 1974;37:668-87.

32. Wessberg GA, LaBanc JP, Epker BN. Musculoskeletal adaptations to surgical advancement of the mandible. Int J Oral Surg 1981;10:417-22.

33. Epker BN, Wessberg GA. Mechanisms of early skeletal relapse following surgical advancement of the mandible. Br J Oral Surg 1982;20:175-82.

34. Epker BN, Wolford LM. Dentofacial deformities. SurgicalOrthodontic correction. St. Louis: The C. V. Mosby Company, 1980.

35. Wessberg GA, Schendel SA, Epker BN. The role of suprahyoid myotomy in surgical advancement of the mandible via sagittal split ramus osteotomies. J Oral Surg 1982;40:273-7.

36. Schendel SA, Epker BN. Results after mandibular advancement surgery: an analysis of 87 cases. J Oral Surg 1980;38:265-82.

\section{Reprint requests to:}

Dr. David S. Carlson

Department of Orthodontics, School of Dentistry,

The University of Michigan,

Ann Arbor, MI 48109-1078 